\title{
Síntomas psicopatológicos en pacientes afectos de cefalea crónica con o sin fibromialgia
}

\author{
I. Sala ${ }^{\text {a,e }}$, C. Roig ${ }^{\text {b }}$ J.A. Amador-Campos ${ }^{\text {e }}$, \\ C. García-Sánchez ${ }^{\text {a }}$ A. Rodríguez ${ }^{\text {c }}$, C. Díaz ${ }^{\text {c }}$, I. Gich $^{\text {d }}$
}

\begin{abstract}
SÍNTOMAS PSICOPATOLÓGICOS EN PACIENTES AFECTOS DE CEFALEA CRÓNICA CON O SIN FIBROMIALGIA
Resumen. Introducción. La presencia de sintomatología psicopatológica, como la ansiedad y la depresión, en las cefaleas crónicas y en la fibromialgia es común. Objetivos. Estudiar si existen diferencias en el perfil psicopatológico entre pacientes con cefalea crónica y fibromialgia (CCFM) y pacientes con cefalea crónica sin fibromialgia (CC), y si existen diferencias en la respuesta al tratamiento entre ambos grupos. Pacientes y métodos. Se administra una batería de test de evaluación de síntomas psicopatológicos a 30 pacientes con CC y a 30 pacientes con CCFM diagnosticados por un reumatólogo. Incluimos en la CC a pacientes con migraña crónica y cefalea tensional crónica. El diseño es con datos apareados de edad y sexo. Se comparan las puntuaciones de depresión, ansiedad, obsesión y perfil de sintomatología psicopatológica -inventario multifásico de personalidad de Minnessota (MMPI-2) y cuestionario de 90 síntomas revisado (SCL-90-R)- (prueba $\mathrm{t}$ ). Se correlacionan los datos con la respuesta al tratamiento. Resultados. Los pacientes con CCFM muestran puntuaciones significativamente superiores en las escalas de hipocondriasis, depresión, histeria, paranoia, psicastenia y esquizofrenia (MMPI-2), y más somatizaciones, obsesión y ansiedad según el SCL-90-R. La respuesta favorable al tratamiento es inferior en el grupo CCFM $(17,85 \%)$ que en el CC (42,85\%). La hipocondriasis se correlaciona con una peor respuesta (regresión logística). Conclusiones. Los pacientes con CCFM muestran un perfil psicopatológico con mayores puntuaciones y síntomas que los pacientes sin fibromialgia. Esta diferencia se correlaciona con la respuesta terapéutica. [REV NEUROL 2009; 49: 281-7]

Palabras clave. Cefalea crónica. Fibromialgia. Hipocondriasis. Respuesta terapéutica. Síntomas psicopatológicos. Tratamiento farmacológico.
\end{abstract}

\section{INTRODUCCIÓN}

La cefalea crónica (CC) y la fibromialgia son dos enfermedades con muchos aspectos comunes. La cefalea crónica se define, según la Sociedad Internacional de Cefaleas (IHS) [1], como dolor de cabeza más de 15 días al mes durante más de tres meses, y se clasifica en migraña crónica, cefalea de tensión crónica, hemicránea continua y cefalea crónica desde el inicio, todas ellas, con o sin abuso de fármacos. Por otro lado, para el diagnóstico de fibromialgia, según el Colegio Americano de Reumatología (ACR) [2], es necesaria la presencia de historia de dolor generalizado bilateral por encima y debajo de la cintura, al menos de tres meses de evolución, y dolor a la palpación digital, con una fuerza aproximada de $4 \mathrm{~kg}$, en 11 o más de los 18 puntos fibromiálgicos entre los dos lados del cuerpo (cervical bajo, segunda costilla, epicondíleo, rodilla, occipital, trapezoidal, supraespinoso, glúteo, trocantéreo).

El síntoma principal que caracteriza a estas enfermedades es el dolor crónico, en el primer caso centrado en la región cefálica y en el segundo más generalizado y difuso por todo el cuerpo; las dos también presentan síntomas asociados parecidos, en-

\footnotetext{
Aceptado tras revisión externa: 12.01.09.

${ }^{a}$ Unidad de Neuropsicología. ${ }^{b}$ Departamento de Neurología. ${ }^{c}$ Sección de Reumatología. ${ }^{d}$ Servicio de Epidemiología Clínica. Hospital de la Santa Creu i Sant Pau. Universitat Autònoma de Barcelona. ${ }^{e}$ Departamento de Personalidad, Evaluación y Tratamientos Psicológicos. Facultad de Psicología. Universitat de Barcelona. Barcelona, España.

Correspondencia: Dr. Carles Roig Arnall. Servicio de Neurología. Departamento de Neurología. Hospital de la Santa Creu i Sant Pau. Universitat Autònoma de Barcelona. Sant Antoni M. Claret, 167. E-08025 Barcelona. Fax: +34935 565 602. E-mail: croig@santpau.cat

Trabajo presentado como comunicación oral en la LIX Reunión Anual de la Sociedad Española de Neurología.

(c) 2009, REVISTA DE NEUROLOGÍA
}

tre ellos, síntomas de ansiedad y de depresión. La prevalencia de la CC es del $4-5 \%$ en población general $[3,4]$, y la de la fibromialgia es del $3 \%$ [5,6], predominantemente en mujeres adultas. La incidencia de la fibromialgia es de 6 hombres y de 11 mujeres por 1.000 habitantes al año [7]. La etiología de ambas enfermedades es desconocida y con síntomas asociados parecidos, entre ellos, síntomas de ansiedad y de depresión.

Diversos estudios muestran que del 24 al $48 \%$ de pacientes con migraña transformada o cefalea crónica diaria presentan fibromialgia [8-11], una proporción significativamente superior al grupo control sin cefalea, en el que sólo el $5 \%$ tiene fibromialgia [12], y claramente más prevalente en mujeres [13]. Cuando se analiza de forma inversa, se observa que del 76 al $100 \%$ de población con fibromialgia tiene algún tipo de cefalea según los criterios diagnósticos de la IHS $[11,14]$.

Existen muy pocos estudios que evalúen la situación psicopatológica de los pacientes afectos de cefalea crónica y fibromialgia. El 57\% de los pacientes con las dos patologías presenta ansiedad, el $39 \%$ depresión, el 55\% síntomas obsesivos, el $71 \%$ somatizaciones y el $47 \%$ alexitimia [15]. Se ha objetivado que las diferencias en el perfil psicopatológico, evaluado con el cuestionario de 90 síntomas, entre un grupo con migraña episódica y fibromialgia o sólo con fibromialgia son estadísticamente significativas en las escalas de somatizaciones, obsesióncompulsión, depresión, ansiedad fóbica, hostilidad y psicoticismo; en todos los casos, el grupo de migraña episódica más fibromialgia presentaba puntuaciones significativamente superiores [13].

El objetivo principal del presente trabajo es estudiar el perfil psicopatológico de pacientes con cefalea crónica y fibromialgia (CCFM) y pacientes con CC sin fibromialgia. El objetivo secundario es analizar la posible influencia del perfil psicopatológico en la respuesta al tratamiento. 


\section{PACIENTES Y MÉTODOS Muestra}

La muestra está formada por 60 pacientes ( 58 mujeres) divididos en dos grupos: 30 con $\mathrm{CC}$ diagnosticada según los criterios de la IHS [1] y 30 con CCFM diagnosticada según los criterios del ACR [2]. El grupo de pacientes con CC está constituido por 14 con migraña crónica y 16 con cefalea tensional crónica. En el grupo de pacientes con CCFM, seis tienen migraña crónica y 24 cefalea tensional crónica. La media de edad es de 55,5 \pm 7,7 años. Para la muestra total, la media de escolarización es de $10 \pm 8,6$ años. No existen diferencias significativas entre los dos grupos en edad $(t=0,07$; $p=0,95)$ ni en años de escolaridad $(t=0,61$; $p=0,48$ )

Se clasifican como abuso de medicación a aquellos pacientes que utilizan analgésicos, ergóticos o triptanes más de la mitad de los días durante tres meses o más [1], sin presuponer que la cefalea esté causada por el abuso.

En la tabla I se recogen las características de la muestra.

Se ha tratado farmacológicamente a los pacientes de acuerdo con las guías oficiales de la Sociedad Española de Neurología [16]. En todos los casos, junto con el tratamiento preventivo, se intentó el cambio de la medicación aguda, y se pasó de analgésicos opiáceos y de ergotamina a analgésicos simples o antiinflamatorios no esteroideos. La figura 1 muestra la frecuencia de los distintos tratamientos farmacológicos que se administraron.

Se realizó un seguimiento de los pacientes a los tres y seis meses para conocer la respuesta al tratamiento. El paciente registraba, a lo largo de este período, en un calendario, la frecuencia de días de dolor de cabeza. Se consideraba buena respuesta si había una disminución del 50\% o más del número de días de cefalea al mes. El grupo de no respuesta está formado por los pacientes que no presentaban mejora a los tres ni a los seis meses. El grupo de respuesta favorable está formado por aquéllos en los que se observaba una reducción de la frecuencia de días de cefalea a los tres meses y se confirmaba la disminución del 50\%, como mínimo, a los seis meses, y por los pacientes que mejoraban al menos el $50 \%$ de días de cefalea a los tres meses y persistía la mejora a los seis meses. En cuatro casos no se pudo valorar la respuesta (tres no volvieron a la visita de seguimiento y uno tuvo una respuesta espontánea sin tratamiento).

La tabla II muestra los días de cefalea en la visita inicial y después del tratamiento para los dos grupos de respuesta.

\section{Instrumentos}

El inventario de ansiedad estado-rasgo [17] es un autoinforme que valora ansiedad estado y rasgo. El tiempo de administración no sobrepasa los 15 minutos. En este estudio sólo se administra la escala de ansiedad estado, que se utiliza para determinar los niveles actuales (en el momento de la administración) de la intensidad de la ansiedad como estado emocional transitorio del organismo, que se caracteriza por sentimientos subjetivos de tensión percibidos de forma consciente; es variable en el tiempo, puede fluctuar en intensidad y hace referencia a un momento determinado, a diferencia de la ansiedad rasgo. La escala de ansiedad estado consta de 20 frases que hacen referencia a cómo se puede sentir la persona 'en ese momento'. Cada ítem se evalúa en una escala que va de 0 a 3 puntos $(0=$ nada; $1=$ algo; 2 = bastante; $3=$ mucho). La puntuación pueden variar desde de 0 puntos hasta 60. La consistencia interna de la escala estado varía entre el 0,9 y 0,93 , y la fiabilidad dos mitades es de 0,94 . La validez concurrente con el factor de segundo orden ansiedad del cuestionario de personalidad 16PF de Cattell se encuentra entre 0,55 y 0,58 .

El cuestionario de depresión de Beck [18] es un autoinforme que evalúa la presencia e intensidad de síntomas depresivos. El tiempo de administra-
Tabla II. Medias \pm desviaciones estándares de los días de cefalea pre y postratamiento para los dos grupos de respuesta.

\begin{tabular}{lcc}
\hline & \multicolumn{2}{c}{ Días de cefalea } \\
\hline Grupo de respuesta & Pretratamiento & Postratamiento \\
\hline Sí & $24,04 \pm 6,31$ & $7,24 \pm 8,20$ \\
\hline No & $26,44 \pm 5,84$ & $19,79 \pm 8,52$ \\
\hline
\end{tabular}

ción es de unos 10 minutos, aproximadamente. Consta de 21 ítems que hacen referencia a aspectos particulares de la experiencia y sintomatología de la depresión (por ejemplo, estado de ánimo, sentimientos de fracaso, sueño, hambre). Cada ítem tiene cuatro posibles respuestas, que puntúan en una escala de 0 a 3 puntos, en función del grado de gravedad que el paciente percibe de sus síntomas depresivos. El rango de puntuación oscila entre 0 y 63. Existen unos rangos de puntuación [19] que permiten clasificar la sintomatología como ausencia de depresión, depresión leve, moderada o grave. Muestra una buena consistencia interna $(\alpha$ de Cronbach $=0,76-0,95)$ y la fiabilidad test-retest oscila alrededor de $r=0,8$.

El inventario de obsesiones-compusiones de Maudsley [20] es una escala de autoaplicación desarrollada para explorar el tipo y expansión de quejas obsesivo-compulsivas. No se creó con la intención de que fuera un instrumento diagnóstico, sino para que fuera útil para la búsqueda de la tipología de pacientes obsesivo-compulsivos. El tiempo de administración es de unos cinco minutos de promedio. De los 32 ítems que contiene, los dos primeros son para asegurar que el participante ha entendido la manera de contestar. El resto de ítems puntúan para cuatro aspectos diferentes: comprobación (9), limpieza (11), lentitud-repetición (7) y duda (7). La forma de respuesta es dicotómica (verdadero/falso). Con la suma de todos ellos se obtiene la puntuación total, que puede ir de 0 a 30; cuanto mayor es la puntuación, más presencia de síntomas obsesivocompulsivos. La consistencia interna es de 
Tabla III. Medias \pm desviaciones estándares de las puntuaciones de los pacientes con CC y CCFM en los instrumentos que evalúan síntomas psicopatológicos. Contrastes entre grupos: valores $t$ y significación.

\begin{tabular}{|c|c|c|c|c|c|}
\hline & \multicolumn{2}{|c|}{ Grupo diagnóstico } & \multirow[b]{2}{*}{$t$} & \multirow[b]{2}{*}{$\mathrm{gl}$} & \multirow[b]{2}{*}{$p$} \\
\hline & $\mathrm{CC}$ & CCFM & & & \\
\hline$\overline{\text { STAI }}{ }^{a}$ & $28,9 \pm 11,2$ & $31,3 \pm 14,9$ & 0,712 & 58 & 0,480 \\
\hline $\mathrm{BDI}{ }^{\mathrm{a}}$ & $15,2 \pm 10,7$ & $20,4 \pm 12,0$ & 1,75 & 58 & 0,085 \\
\hline $\mathrm{MOCl}^{\mathrm{a}}$ & $8,8 \pm 5,8$ & $10,2 \pm 5,2$ & 1,02 & 58 & 0,312 \\
\hline \multicolumn{6}{|l|}{ MMPI- $2^{b}$} \\
\hline Sinceridad & $60,2 \pm 10,1$ & $56,5 \pm 10,62$ & 1,35 & 58 & 0,181 \\
\hline Fiabilidad & $55,2 \pm 12,4$ & $59,6 \pm 13,3$ & 1,34 & 58 & 0,185 \\
\hline Actitud & $49,8 \pm 8,9$ & $49,0 \pm 9,5$ & 0,32 & 58 & 0,750 \\
\hline Hipocondriasis & $69,5 \pm 21,0$ & $80,9 \pm 11,8$ & 3,70 & 58 & 0,001 \\
\hline Depresión & $62,13 \pm 12,7$ & $70,9 \pm 10,7$ & 2,88 & 58 & 0,005 \\
\hline Histeria & $63,0 \pm 13,3$ & $75,2 \pm 13,9$ & 3,44 & 58 & 0,001 \\
\hline Desviación psicopática & $50,9 \pm 10,2$ & $51,4 \pm 11,3$ & 0,20 & 58 & 0,839 \\
\hline Masculinidad-feminidad & $50,3 \pm 11,5$ & $46,4 \pm 7,8$ & 1,55 & 58 & 0,125 \\
\hline Paranoia & $51,1 \pm 10,7$ & $58,0 \pm 11,7$ & 2,35 & 58 & 0,021 \\
\hline Psicastenia & $55,6 \pm 11,9$ & $63,4 \pm 13,6$ & 2,33 & 58 & 0,023 \\
\hline Esquizofrenia & $55,7 \pm 13,4$ & $65,9 \pm 13,4$ & 2,92 & 58 & 0,005 \\
\hline Manía & $46,9 \pm 10,4$ & $50,4 \pm 9,5$ & 1,34 & 58 & 0,186 \\
\hline Introversión social & $57,7 \pm 8,9$ & $60,2 \pm 11,4$ & 0,93 & 58 & 0,355 \\
\hline \multicolumn{6}{|l|}{ SCL-90-R ${ }^{b}$} \\
\hline Somatizaciones & $60,8 \pm 10,0$ & $70,1 \pm 7,1$ & 3,76 & 51 & 0 \\
\hline Obsesión-compulsión & $58,8 \pm 11,7$ & $65,5 \pm 7,6$ & 2,37 & 51 & 0,021 \\
\hline Sensibilidad interpersonal & $57,1 \pm 11,3$ & $61,0 \pm 11,5$ & 1,23 & 51 & 0,222 \\
\hline Depresión & $58,3 \pm 11,6$ & $62,8 \pm 10,1$ & 1,46 & 51 & 0,148 \\
\hline Ansiedad & $56,5 \pm 9,8$ & $63,0 \pm 9,5$ & 2,39 & 51 & 0,020 \\
\hline Hostilidad & $51,0 \pm 10,9$ & $54,8 \pm 12,0$ & 1,20 & 51 & 0,234 \\
\hline Ansiedad fóbica & $50,3 \pm 13,8$ & $53,1 \pm 15,2$ & 0,69 & 51 & 0,488 \\
\hline Ideación paranoide & $57,8 \pm 10,3$ & $55,2 \pm 11,8$ & 0,84 & 51 & 0,403 \\
\hline Psicoticismo & $57,5 \pm 11,3$ & $62,0 \pm 11,1$ & 1,43 & 51 & 0,159 \\
\hline Índice de gravedad global & $59,37 \pm 11,51$ & $65,4 \pm 8,07$ & 2,16 & 51 & 0,035 \\
\hline Número de síntomas positivos & $60,2 \pm 11,14$ & $65,7 \pm 8,85$ & 1,97 & 51 & 0,054 \\
\hline Índice de síntomas positivos de distrés & $52,9 \pm 12,37$ & $60,8 \pm 10,82$ & 2,44 & 51 & 0,018 \\
\hline
\end{tabular}

BDI: cuestionario de depresión de Beck; CC: cefalea crónica; CCFM: cefalea crónica con fibromialgia; MMPI-2: inventario multifásico de personalidad de Minnessota; MOCl: inventario de obsesiones-compusiones de Maudsley; SCL-90-R: cuestionario de 90 síntomas revisado; STAI: inventario de ansiedad estado-rasgo. ${ }^{\text {a }}$ Puntuaciones directas; ${ }^{\text {b }}$ Puntuaciones típicas.

rencia a la salud, sensibilidad, familia, hábitos ocupacionales, educación, actitudes, fobias, estado de ánimo, etc. Es aplicable a personas de 16 años o más, con un nivel cultural mínimo de ocho años de escolarización (2. ${ }^{\circ}$ de Enseñanza Secundaria Obligatoria, ESO). Proporciona puntuaciones en diferentes escalas: tres de validez, 10 clínicas, 15 de contenido y 18 suplementarias. En este trabajo sólo se utilizan las escalas de validez: escala de sinceridad (L), escala de fiabilidad $(\mathrm{F})$ y escala de defensividad $(\mathrm{K})$; y las clínicas: hipocondriasis (Hs), depresión (D), histeria (Hy), desviación psicopática (Pd), masculinidad-feminidad (Mf), paranoia (Pa), psicastenia $(\mathrm{Pt})$, esquizofrenia $(\mathrm{Sz})$ manía (Ma) e introversión social (Si).

La forma de respuesta es dicotómica (verdadero/falso). Las puntuaciones directas de cada escala se transforman en puntuaciones típicas de media 50 y desviación típica 10. La interpretación se basa en las puntuaciones típicas obtenidas en cada una de las escalas, en las que destacan aquéllas que muestran puntuaciones significativamente elevadas, y en la configuración general del perfil. Una puntuación típica por encima de 65 indica la presencia de síntomas psicopatológicos dentro de un rango clínico. Clásicamente, la elevación en las escalas de hipocondriasis, depresión e histeria muestra un perfil neurótico; si predomina una elevación en las escalas de paranoia, psicastenia y esquizofrenia (a veces apoyadas por la depresión y la manía), se hablará de un patrón tipo psicótico.

Los autores [25] informan del índice de fiabilidad en seis escalas, que va de 0,52 a 0,83 . La fiabilidad de la adaptación española, evaluada mediante el índice $\alpha$ de Cronbach, es de 0,78 para las diez escalas clínicas. El manual de la adaptación española no proporciona datos sobre la validez.

El cuestionario de 90 síntomas revisado (SCL-90-R) [26] es un autoinforme que permite valorar alteraciones psicopatológicas o psicosomáticas en diferentes dimensiones: somatizaciones (SOM), obsesión-compulsión (OBS), sensibilidad interpersonal (INT), depresión (DEP), ansiedad (ANS), hostilidad (HOS), ansiedad fóbica (FOB), ideación paranoide (PAR) y psicoticismo (PSI). El tiempo de administración es de 12 a 15 minutos. Consta de 90 ítems con respuesta en una escala (tipo Likert) graduada de 0 (ausencia de molestias relacionadas con los síntomas) a

0,77 [21]. El índice de fiabilidad ( $\alpha$ de Cronbach) que se obtiene es de 0,61 $[22,23]$. La validez concurrente con otra prueba que evalúa síntomas de obsesión (Leyton Obsessional Inventory) es de 0,6 [24].

El inventario multifásico de personalidad de Minnessota (MMPI-2) [25] es un cuestionario de evaluación de diferentes dimensiones de la personalidad y de sintomatología psicopatológica. El tiempo que se tarda en cumplimentarlo oscila entre 60 y 90 minutos. Consta de 567 ítems que hacen refe-
4 (molestia máxima) según la intensidad del sufrimiento. Además de las nueve dimensiones sintomáticas, se obtiene una medida generalizada de la intensidad del sufrimiento psíquico y psicosomático global (GSI), un índice que suma el número total de síntomas presentes para conocer la amplitud y diversidad de la psicopatologia (PST), y un índice que relaciona el sufrimiento o 'distrés' global con el numero de síntomas y que sirve de indicador de la intensidad sintomática media (PSDI). 
Las puntuaciones directas se transforman en puntuaciones típicas normalizadas, con media 50 y desviación típica 10 , de manera que se consideran dentro de la normalidad las puntuaciones entre 40 y 60 .

La fiabilidad, evaluada mediante la consistencia interna, oscila entre 0,81 y 0,88 (correlación dos mitades), y entre 0,81 y 0,9 ( $\alpha$ de Cronbach). La correlación test-retest, con un intervalo de una semana, en una muestra psiquiátrica de 219 pacientes, ofrece coeficientes de correlación que varían entre 0,78 y 0,9 [26].

La validez concurrente con las escalas del MMPI, con el inventario de depresión de Beck y con el inventario de ansiedad estado-rasgo de Spielberger es buena. La prueba también presenta una adecuada validez de criterio y se ha demostrado su sensibilidad para apreciar los cambios en contextos clínicos [26,27].

\section{Procedimiento}

Los pacientes reclutados para este estudio acudían por primera vez a la unidad de cefaleas del servicio de neurología por dolor de cabeza. Los exploraba un neurólogo experto en cefaleas que los diagnosticaba según los criterios de la IHS [1]. El grupo de pacientes con fibromialgia lo diagnosticaban reumatólogos especialistas según los criterios ACR [2]. Un psicólogo les administraba la batería de test seleccionada para la evaluación de síntomas de ansiedad, depresión, obsesión y alteraciones psicopatológicas.

Se hacía un seguimiento de la respuesta al tratamiento a los tres y seis meses, tal como se ha especificado en el apartado anterior.

\section{RESULTADOS}

La tabla III recoge las puntuaciones medias y las desviaciones típicas de los dos grupos de pacientes en las diferentes pruebas que evalúan sintomatología psicopatológica. Los contrastes, mediante la prueba $t$ para muestras independientes, ponen de manifiesto que existen diferencias estadísticamente significativas entre el grupo de CC y el grupo de CCFM en las escalas de hipocondriasis, depresión, histeria, paranoia, psicastenia y esquizofrenia del MMPI-2. También se han encontrado diferencias significativas entre ambos grupos en las escalas de somatizaciones, obsesiones, ansiedad, el índice de gravedad global y el índice de síntomas positivos de distrés del SCL-90-R. En todos los casos, los pacientes con CCFM son los que obtienen las puntuaciones más elevadas. Las figuras 2 y 3 recogen los perfiles, en puntuaciones típicas, de los pacientes en el MMPI-2 y en el SCL-90-R.

Para estudiar las variables que predicen mejor la respuesta al tratamiento, se ha realizado una regresión logística por pasos (foward step), en la que la variable dependiente es la respuesta al tratamiento, y como variables independientes se han incluido: el grupo (CC, CCFM), la edad, la presencia de abuso farmacológico (sí/no), el tipo de tratamiento (con psicotropos o no), y las puntuaciones en las escalas de los test que evalúan síntomas psicopatológicos. La tabla IV recoge los resultados de la regresión logística.

De todas las variables introducidas, las variables seleccionadas han sido: grupo (paso 1) e hipocondriasis (paso 2). Los valores de la odds ratio muestran que pertenecer al grupo CC multiplica por seis las posibilidades de obtener una buena respuesta al tratamiento. Además, para el grupo CCFM, cada aumento de un punto en la escala de hipocondriasis del MMPI-2 disminuye en un $10 \%$ la probabilidad de obtener una buena respuesta al tratamiento.

Finalmente, para evaluar el rendimiento del modelo, se ha elaborado una nueva variable, que se ha denominado pronóstico, combinando el grupo (CC y CCFM) y la puntuación en la escala de hipocondriasis del MMPI-2.
Con esta nueva variable se ha realizado una curva ROC para conocer la probabilidad de una buena o mala respuesta al tratamiento. Los resultados muestran un área bajo la curva de 0,859 (intervalo de confianza al 95\%= 0,752-0,96). Con una puntuación de 0,48 en la variable pronóstico, el índice de sensibilidad es del 85,3, y el índice de especificidad, del 81,8 (Fig. 4).

Los pacientes que pertenecen al grupo CC, independientemente de la puntuación que tengan en hipocondriasis, es probable que obtengan una buena respuesta al tratamiento. Los pacientes que además de CC tengan fibromialgia (CCFM), si muestran una puntuación típica superior a 75 en la escala de hipocondriasis del MMPI-2, tendrán más riesgo de presentar una mala respuesta al tratamiento.

\section{DISCUSIÓN}

La presencia de síntomas psicopatológicos en pacientes con CC está ampliamente documentada. Los síntomas de ansiedad y depresión están presentes tanto en las migrañas crónicas $(80 \%)$ como en las cefaleas tensionales crónicas (90\%) [28-33]. También es frecuente la presencia de un perfil psicopatológico más alterado, evaluado con el MMPI-2 [34-37] o con el SCL-90-R [38] respecto a los controles sin cefalea.

En el caso de la fibromialgia, los estudios de comorbilidad psiquiátrica muestran mayor presencia de sintomatología de- 


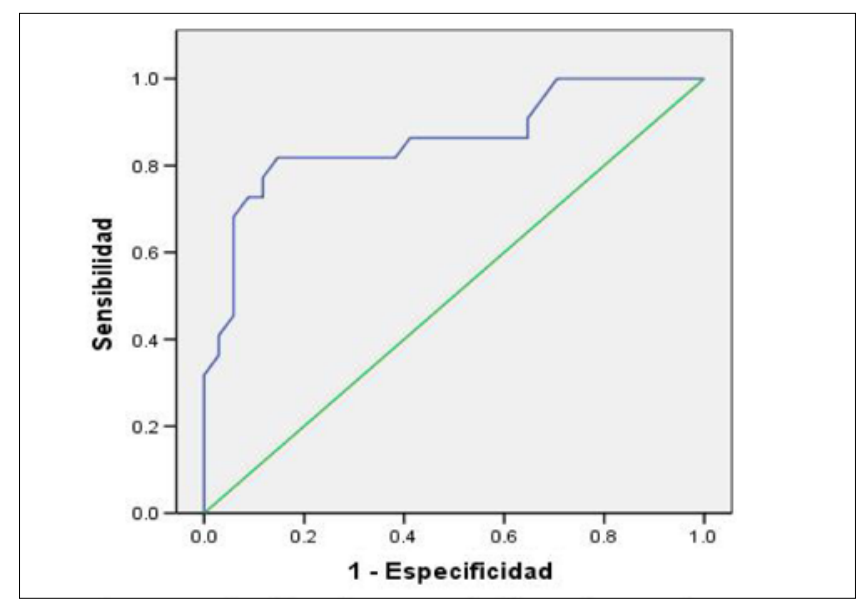

Figura 4. Gráfica de la curva ROC para la variable pronóstico (los segmentos diagonales son producidos por los empates).

Tabla IV. Resultados de la regresión logística.

\begin{tabular}{lllll}
\hline Variables de la ecuación & Significación & OR & \multicolumn{2}{c}{ IC 95\% OR } \\
\cline { 3 - 5 } & & & Inferior & Superior \\
\hline Grupo (1) & 0,011 & 6,4 & 1,54 & 26,63 \\
\hline Hipocondriasis (MMPI-2) & 0,010 & 0,912 & 0,85 & 0,97 \\
\hline Hosmer y Lemeshow & 0,177 & & & \\
\hline IC 95\%: intervalo de confianza al 95\%; OR: odds ratio. & & \\
\hline
\end{tabular}

presiva y trastornos de ansiedad respecto a sujetos sin fibromialgia [39-41]. En comparación con un grupo control sin fibromialgia, los pacientes con fibromialgia presentan puntuaciones significativamente superiores en hipocondriasis, histeria y psicastenia; en la tríada neurótica (hipocondriasis, depresión e histeria) o en el perfil conversivo (hipocondriasis e histeria) evaluado con el MMPI [42]. Si se comparan pacientes con fibromialgia y pacientes con artritis, se observan diferencias en el índice de distrés y en las escalas de somatizaciones, depresión y psicoticismo del SCL-90-R [43,44], aunque algunos estudios no encuentran diferencias entre los dos grupos [45].

Como se ha comentado antes, existen pocos trabajos, hasta el momento, que evalúen la situación psicopatológica de los pacientes afectos de CCFM. El objetivo principal de este trabajo es estudiar el perfil psicopatológico de pacientes con CCFM y pacientes con CC. Los pacientes con CCFM presentan puntuaciones significativamente más elevadas que los pacientes afectos sólo de $\mathrm{CC}$ en los test que evalúan sintomatología psicopatológica.

El perfil psicopatológico del grupo de pacientes con $\mathrm{CC}$ se caracteriza por la presencia de síntomas de ansiedad y depresión leves, síntomas de hipocondriasis elevados, con puntuaciones que alcanzan dos desviaciones típicas por encima de la media, y una puntuación relativamente elevada, pero dentro de la normalidad, en la escala de somatizaciones del SCL-90-R. Estos resultados sugieren la presencia de múltiples quejas somáticas e importantes preocupaciones por la propia salud.

En cuanto al grupo formado por pacientes con CCFM, se observa un perfil con síntomas de ansiedad y depresión moderados, y un perfil psicopatológico con puntuaciones elevadas en hipocondriasis, depresión, histeria y esquizofrenia del MMPI-2, y en la escala de somatizaciones del SCL-90-R. Los pacientes con CCFM muestran la presencia de distintos síntomas, como tristeza, falta de motivación y de energía, aumento de síntomas físicos en situaciones de elevada tensión, preferencia por las actividades en solitario, sentimientos de soledad y alienación social. Predominan los síntomas de hipocondriasis, como la preocupación por la salud física y la manifestación de diferentes síntomas físicos.

En general, el grupo CCFM presenta más quejas somáticas, más síntomas de depresión, más sensibilidad emocional, más ansiedad y más síntomas de alienación social que el grupo que sólo tiene CC.

Estos resultados son semejantes a los hallados por otros autores, que encuentran más sintomatología psicopatológica y malestar en los pacientes que, además de cefalea, tienen fibromialgia $[6,13]$, y coinciden en hallar puntuaciones significativamente elevadas en las escalas de hipocondriasis del MMPI y de somatizaciones del SCL-90-R, ya que ambas miden las presencia de síntomas físicos, como dolor de cabeza, dolor de espalda, trastornos digestivos, cansancio, debilidad, hormigueo en las manos, ataques de náuseas o vómitos, dolores en el pecho, etc.

Estos datos sugieren que existe una estrecha relación entre los sistemas del dolor y los de regulación emocional, que podría explicar la patogenia de las dos entidades a partir de un proceso de sensibilización central. Algunos estudios han encontrado marcadores biológicos comunes en las dos patologías como un nivel más elevado de glutamato en el fluido cerebroespinal, en pacientes con migraña crónica y fibromialgia, respecto a los que no tienen fibromialgia o a un grupo control [46], que correlaciona de forma significativa con niveles elevados del factor de crecimiento neuronal y factor neurotrófico cerebral, tanto en pacientes con migraña crónica como en pacientes con fibromialgia [47]. También el mecanismo de la serotonina se encuentra relacionado tanto en la cefalea como en la fibromialgia, y muestra eficacia tras el tratamiento con antidepresivos [48] o con una combinación de antiepilépticos con antidepresivos [49]. Aunque aproximadamente el $40 \%$ de los pacientes que presentan cefalea muestra una hiperalgesia generalizada, parece que existe una alteración en el mecanismo central de modulación del dolor [50].

En relación con el segundo objetivo, los resultados obtenidos permiten afirmar que en la muestra de este estudio, tener fibromialgia y una puntuación significativamente elevada en hipocondriasis predice una mala respuesta al tratamiento farmacológico de las CC. No existen estudios previos que hayan analizado la relación entre los síntomas psicopatológicos y la respuesta al tratamiento en pacientes con CCFM.

En resumen, los pacientes con CCFM presentan un perfil psicopatológico similar a los pacientes con CC sin fibromialgia, pero con puntuaciones globalmente superiores y un porcentaje menor de respuesta al tratamiento. También hemos encontrado una relación significativa entre la pertenencia al grupo CCFM y mayor puntuación en hipocondriasis con la peor respuesta a los tratamientos farmacológicos.

Los autores que han estudiado la evolución de la fibromialgia han observado una percepción de mejora subjetiva en el ámbito cognitivo y una mayor satisfacción en salud general, seguramente debidas a un proceso de adaptación a la presencia continuada de los síntomas. Sin embargo, también observan que casi la mitad de los pacientes presenta los mismos síntomas, y un pequeño porcentaje muestra mejora a largo plazo [51,52]. 
La hipocondriasis o la preocupación excesiva por la salud es un fenómeno frecuente tanto en individuos sanos como enfermos. Según Avia [53], los pacientes hipocondríacos presentan quejas, desproporcionadas y variables, de síntomas físicos y muestran más preocupación por su posible significación y etiología que por el dolor que les causan. Refieren gran temor a la enfermedad e importante preocupación por sus funciones vitales y fisiológicas, en ocasiones con auténtica convicción de estar enfermos y cerca de la muerte. En ocasiones, dudan de los resultados negativos de las pruebas que se les practican e incluso de la opinión del profesional médico.

La proporción de pacientes con actitudes hipocondríacas y síntomas funcionales puede variar según el instrumento de medida utilizado [53]. La escala de hipocondriasis del MMPI se ha utilizado en diferentes estudios como un indicador clínico de la hipocondría. Esta escala contiene ítems que detectan la presencia de diversos síntomas físicos, un elemento relevante en el síndrome, pero no valora las actitudes hipocondríacas, otro elemento importante de la enfermedad. Barsky et al [54] realizaron un análisis de regresión múltiple, utilizando como variables dependientes las actitudes hipocondríacas y los síntomas somáticos, y como variables predictoras la edad, el sexo, la posición social, el estado civil, el diagnóstico médico, la puntuación en depresión en el cuestionario de Beck, la utilización total de servicios médicos y la historia psiquiátrica. El predictor más importante, tanto de las actitudes hipocondríacas como de los síntomas somáticos, fue el grado de depresión, que explicó el 33\% de las variaciones de las primeras y el $26 \%$ de los segundos. Los síntomas somáticos son el principal motivo de consulta y la ra- zón principal por la que los pacientes con trastornos mentales como la ansiedad y la depresión acuden inicialmente a atención primaria [55].

La hipocondría está fuertemente relacionada con la ansiedad y los trastornos afectivos. Se considera que es la consecuencia de la existencia de un estrés general, aunque el motivo principal de preocupación en estos pacientes sean los síntomas físicos y, por lo tanto, tienden a acudir al médico en lugar del psicólogo o al psiquiatra [44]. Otra explicación que se ha dado a la hipocondría es la presencia de un proceso de amplificación de diversas sensaciones corporales que están relacionadas con el estado de ánimo; se tiende a una interpretación pesimista de la realidad y a la atribución de consecuencias graves para síntomas físicos leves o moderados. Esto podría explicar la mayor proporción de pacientes hipocondríacos que presentan una peor respuesta al tratamiento. En definitiva, se observa otra vez una interrelación entre factores físicos y emocionales, lo que sugiere una alteración en el procesamiento central del dolor que da lugar a una peor respuesta terapéutica.

Cabe señalar algunas limitaciones de este trabajo. En primer lugar, el tamaño de la muestra; se trata de un grupo reducido, lo que hace que los resultados se deban interpretar con la debida cautela. En segundo lugar, hay que tener en cuenta que la escala de hipocondriasis del MMPI-2 recoge la presencia de síntomas físicos y no de actitudes hipocondríacas, que constituyen uno de los rasgos más importantes de la hipocondría. Finalmente, el tiempo de evolución de la cefalea no lo hemos considerado, debido a la dificultad, en la mayoría de los casos, de precisar con certeza el inicio de la cefalea.

\section{BIBLIOGRAFÍA}

1. Classification Subcommittee of the International Headache Society. The international classification of headache disorders. 2 ed. Cephalalgia 2004; 24 (Suppl 1): 1-160.

2. Wolfe F, Smythe HA, Yunus MB, Bombardier C, Goldenberg DJ, Tugwell P, et al. The American College of Rheumatology 1990 criteria for the classification of fibromyalgia: report of the Multicenter Criteria Committee. Arthritis Rheum 1990; 33: 160-72.

3. Castillo J, Muñoz P, Guitera V, Pascual J. Epidemiology of chronic daily headache in the general population. Headache 1999; 39:190-6.

4. Silberstein SD, Lipton RB, Delessio DJ. Chronic daily headache, including transformed migraine, chronic tension type headache, and medication overuse. In Wolff's headache and other head pain. New York: Oxford University Press; 2001. p. 247-81.

5. Collado A, Alijotas J, Benito P, Alegre A, Romera M, Sañudo I, et al. Documento de consenso sobre el diagnostico de la fibromialgia en $\mathrm{Ca}$ taluña. Med Clin 2002; 118: 745-9.

6. Leza JC. Fibromialgia: un reto también para la neurociencia. Rev Neurol 2003; 36: 1165-75.

7. Weir PT, Harlan GA, Nkoy FL, Jones SS, Hegmann DT. The incidence of fibromialgia and its associated comorbidities: a population-based retrospective cohort study based on International Classification of Diseases, 9th revision codes. J Clin Rheumatol 2006; 12: 124-8.

8. Peres MFP, Young WB, Kaup AO, Zukerman E, Silberstein SD. Fibromyalgia is common in patients with transformed migraine. Neurology 2001; 57: 1326-8.

9. Kapisyzi M, Kapisyzi M, Dobi D, Doka R, Roci E, Basha E, et al. Prevalence of fibromialgia in patients with chronic daily headache. $\mathrm{Ce}$ phalalgia 2007; 27: 679 .

10. Pavia T, Batista A, Martins P, Martins A. The relationship between headaches and sleep disturbances. Headache 1995, 35: 590-5.

11. Nicolodi M, Sarzi-Puttini S, Sicuteri F. Fibromyalgia suffers are headache suffers. Proceedings of the 7th International Headache Congress, Toronto, Canada. September 16-20, 1995. Cephalalgia 1995; 15 (Suppl 14): 294

12. Tommaso M, Lamberti P, Livrea P, Pecoraro C, Prudenzano MP, Sardaro M, et al. Fibromyalgia comorbidity in primary headache. Cephalalgia 2007; 27: 580.

13. Ifergane G, Buskila D, Simiseshvely N, Zeev K, Cohen H. Prevalence of fibromyalgia syndrome in migraine patients. Cephalalgia 2006; 26 : 451-6.

14. Marcus DA, Bernstein C, Rudy TE. Fibromyalgia and headache: an epidemiological study supporting migraine as part of the fibromyalgia syndrome. Clin Rheumatol 2005; 24: 595-601.

15. Centonze V, Bassi A, Cassiano MA, Munno I, Dalfino L, Causarano V. Migraine, daily chronic headache and fibromyalgia in the same patient: an evolutive 'continuum' of non organic chronic pain? About 100 clinical cases. Neurol Sci 2004; 25: S291-2.

16. Comité ad hoc del Grupo de Estudio de la Cefalea. Sociedad Española de Neurología. Guía para el diagnóstico y tratamiento de las cefaleas. Barcelona: Prous; 2006.

17. Spielberger CD, Gorsuch RL, Lushene RE. Cuestionario de ansiedad estado-rasgo. Adaptación española. Madrid: TEA; 2002.

18. Beck AT, Ward CH, Mendelson M, Mock J, Erbaugh J. Inventory for measuring depression. Arch Gen Psychiatr 1961; 4: 561-71.

19. Beck AT, Steer RA, Garbin MC. Psychometric properties of the Beck Depression Inventory. Twenty-five years of evaluation. Clin Psychol Rev 1988; 8: 77-100.

20. Hodgson RJ, Rachman S. Obsessional-compulsive complaints. Behav Res Ther 1977; 15: 389-95.

21. Sanavio E, Vidotto G. The components of the Maudsley ObsessionalCompulsive questionnaire. Behav Res Ther 1985; 23: 659-62.

22. Stoylen IJ, Larsen S, Kvale G. The Maudsley Obsessional-Compulsive Inventory and OCD in a Norwegian nonclinical sample. Scand J Psychol 2000; 41: 283-6.

23. Sternberger LG, Burns GL. Maudsley Obsessional-Compulsive Inventory: obsessions and compulsions in a nonclinical sample. Behav Res Ther 1990; 28: 334-40.

24. Arena JG, Blanchard EB, Andrasik F, Applebaum K. Obsessions and compulsions in three kinds of headache sufferers: analysis of the Maudsley questionnaire. Behav Res Ther 1986; 24: 127-32.

25. Hathaway SR, McKinley JC. El inventario multifásico de personalidad de Minnessota (Minnessota Multiphasic Personality, MMPI-2). Madrid: TEA; 1999.

26. Derogatis LR. SCL-90-R Administration and procedures. Manual II for the revised version of the SCL-90-R. Baltimore: John Hopkins University Press; 1983. 
27. Derogatis LR. SCL-90-R Cuestionario de 90 síntomas. Adaptación española. Madrid: TEA; 2002.

28. Merikangas KR, Angst J, Isler H. Migraine and psychopathology. Arch Gen Psychiatry 1990; 47: 849-53.

29. Breslau N. Psychiatric comorbidity in migraine. Cephalalgia 1998; 18: 56-61.

30. Juang KD, Wuang SJ, Fuh JL, Lu SR, Su TP. Comorbidity of depressive and anxiety disorders in chronic daily headache and its subtypes. Headache 2000; 40: 818-23.

31. Radat F. Psychopathologie et céphalées. Rev Neurol (Paris) 2000; 156: 662-7.

32. Serrano-Dueñas M. Cefalea tipo tensional crónica y depresión. Rev Neurol 2000; 30: 822-6.

33. Cardona-Castrillón GP, Isaza R, Zapata-Soto AP, Franco JG, González-Berrio C, Tamayo-Díaz CP. Comorbilidad de trastorno depresivo mayor, trastorno distímico y trastornos de ansiedad con migraña. Rev Neurol 2007; 45: 272-5.

34. De Benedittis G, Lorenzetti A. Minor stressful life events (daily hassles) in chronic primary headache: relationship with MMPI personality patterns. Headache 1992; 32: 330-2.

35. Inan L, Soykan C, Tulunay FC. MMPI profiles of Turkish headache sufferers. Headache 1994; 34: 152-4.

36. Aguirre J, Gallardo R, Pareja JA, Pérez-Miranda M. Cluster of MMPI personality profiles in chronic tension-type headache and predictable response to fluoxetine. Cephalalgia 2000; 20: 51-6.

37. Mongini F, Ibertis F, Barbalonga E, Raviola F. MMPI-2 profiles in chronic daily headache and their relationship to anxiety levels and accompanying symptoms. Headache 2000; 40: 466-72.

38. Puca FM, Antonaci F, Genco S, Savarese MA, Piazzolla G, Prudenzano MP. Psychologic factors in chronic headache: assessment by means of the SCL-90-R Inventory. Cephalalgia 1989; 9: 33-51.

39. Martin MJ, Luque M, Solé P, Mengual A, Granados J. Aspectos psicológicos de la fibromialgia. Revista de Psiquiatría de la Facultad de Medicina de Barcelona 2000; 27: 12-8.

40. Mañez I, Fenollosa P, Martínez-Azucena A, Salazar A. Revista de la Sociedad Española del Dolor 2005; 12: 491-500.

41. Hudson JI, Goldenberg DL, Pope HC, Keck PE, Schlesinger L. Comorbidity of fibromyalgia with medical and psychiatric disorders. Am J Med 1992; 92: 363-7.

42. Pastor-Mira A, López-Roig S, Rodríguez-Marín J, Jaun-Quilis V. Evo- lución en el estudio de la relación entre factores psicológicos y fibromialgia. Psychothema 1995; 7: 627-39.

43. Uveges JM, Parker JC, Smarr KL, McGowan JF, Lyon MG, Irvin WS, et al. Psychological symptoms in primary fibromyalgia syndrome: relationship to pain, life stress, and sleep disturbance. Arthritis Rheum 1990; 33: 1279-83.

44. Kirmayer LJ, Robbins JM, Kapusta MA. Somatizations and depression in fibromyalgia syndrome. Am J Psychiatry 1988; 145: 950-4.

45. Clark S, Campbell SM, Forehand ME, Tindall EA, Bennett RM. Clinical characteristics of fibrositis. II. A blinded controlled study using standard psychological tests. Arthritis Rheum 1985; 28: 132-7.

46. Peres MF, Zukerman E, Senne-Soares CA, Alonso EO, Santos BF, Faulhaber MH. Cerebrospinal fluid glutamate levels in chronic migraine. Cephalalgia 2004; 24: 735-9.

47. Sarchielli P, Mancini ML, Floridi A, Coppola F, Rossi C, Nardi K, et al. Increased levels of neurotrophins are not specific for chronic migraine: evidence from primary fibromyalgia syndrome. J Pain 2007; 8: 737-45.

48. Nicolodi M, Volpe AR, Sicuteri F. Fibromyalgia and headache. Failure of serotonergic analgesia and N-methyl-D-aspartate-mediated neuronal plasticity: their common clues. Cephalalgia 1998; 18 (Suppl 21): 41-4.

49. Smith NL. Serotonin mechanisms in pain and functional syndromes: management implications in comorbid fibromyalgia, headache, and irritable bowl syndrome-case study and discussion. J Pain Palliat Care Pharmacother 2004; 18: 31-45.

50. Okifujy A, Turk D, Marcus D. Comparison of generalized and localized hiperalgesia in patients with recurrent headache and fibromialgia. Psychosom Med 1999, 61: 771-80.

51. Wolfe F, Anderson J, Harkness D, Bennett RM, Caro XJ, Goldenberg DL, et al. Health status and disease severity in fibromyalgia: results of a six-center longitudinal study. Arthritis Rheum 1997; 40: 1571-9.

52. Baumgartner E, Finckh A, Cedraschi C, Vischer TL. A six year prospective study of a cohort of patients with fibromialgia. Ann Rheum Dis 2002; 61: 644-5.

53. Avia MD. Hipocondría. Barcelona: Martínez Roca; 1993.

54. Barsky A, Wyshak G, Klerman G. Hypocondriasis: an evaluation of the DSM III criteria in medical outpatients. Arch Gen Psychiatry 1986; 43: 493-500.

55. Kroenke K. Patients presenting with somatic complaints: epidemiology, psychiatric comorbidity and management. Int J Methods Psychiatr Res 2003; 12: 34-43.

\section{PSYCHOPATHOLOGICAL SYMPTOMS IN PATIENTS SUFFERING FROM CHRONIC CEPHALEA WITH OR WITHOUT FIBROMYALGIA}

Summary. Introduction. The presence of psychopathological symptoms as anxiety and depression in chronic daily headache and in fibromyalgia is common. Aim. To study whether there are any difference in the psychopathological profile and treatment response between patients with chronic headache and fibromyalgia (CHFM) and patients with chronic headache without fibromyalgia $(\mathrm{CH})$. Patients and methods. A comprehensive psychological test battery was administered to 30 patients with $\mathrm{CH}$ and 30 patients with CHFM, diagnosed by a rheumatologist. We included chronic migraine and chronic tensional headache in CH group. Patients were matched for age and gender. Depression, anxiety and obsession scores, and the profile of psychopathological symptoms (MMPI-2, SCL-90-R) were compared ( $\mathrm{t}-\mathrm{test})$. Correlations between symptoms and treatment response were examined. Results. Patients with CHFM showed significant highest scores on hypochondriasis, depression, hysteria, paranoia, psychasthenia and schizophrenia (MMPI-2) and more somatization, obsession and anxiety according to SCL-90-R. A poorer response to treatment was observed in CHFM (17.85\%) versus $\mathrm{CH}(42,85 \%)$ group. Conclusions. Patients with CHFM showed a psychopathological profile with highest scores and symptoms than patients without fibromyalgia. These differences correlated with the therapeutic response. [REV NEUROL 2009; 49: 281-7]

Key words. Chronic headache. Fibromyalgia. Hipocondriasis. Pharmachological treatment. Psychopathological symptoms. Therapeutic response. 